\title{
Técnica da instilação de colírios em pacientes portadores de glaucoma crônico
}

\author{
Eyedropinstillation technique in chronic glaucoma patients
}

\author{
Paulo Gelman Vaidergorn ${ }^{1}$ \\ Roberto Freire Santiago Malta ${ }^{2}$ \\ Adriana Silva Borges ${ }^{1}$ \\ Maria Denise Rocha Menezes $^{3}$ \\ Eliomar Santana Trindade ${ }^{3}$ \\ João Baptista Nigro Santiago Malta ${ }^{4}$
}

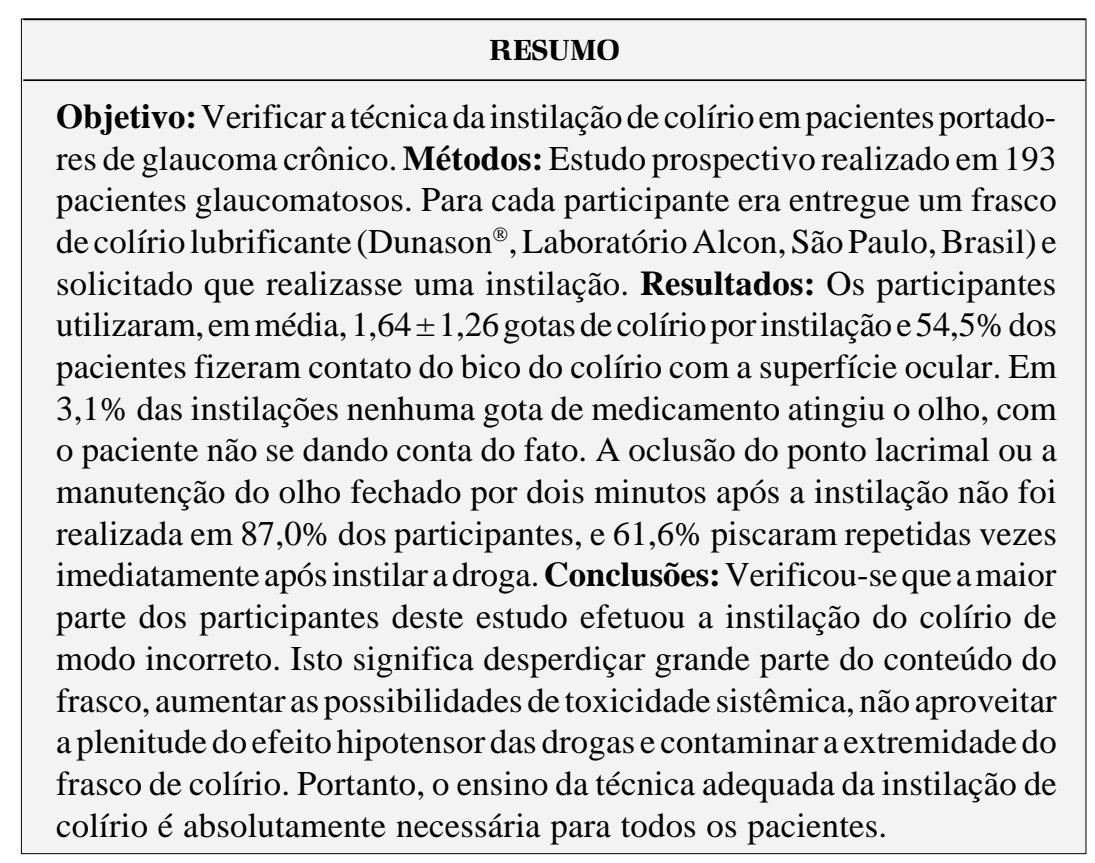

Descritores: Glaucoma/terapia; Soluções oftálmicas; Instilação de medicamentos; Prescrição de medicamentos

\section{INTRODUÇÃO}

Não obstante o enorme progresso científico observado no decorrer do século XX, a maior parte dos doentes portadores de glaucoma ainda é tratada por meio da prescrição de drogas sob a forma de colírios. Para que a terapêutica clínica antiglaucomatosa seja bem sucedida faz-se necessário a cooperação do paciente no sentido de utilizar corretamente a medicação $\operatorname{prescrita}^{(1)}$. Isto inclui, não somente, a aquisição e o uso dos colírios nos horários recomendados, como, também, a técnica de instilá-los corretamente.

É sabido que o glaucoma crônico, por sua própria natureza, induz os pacientes a uma má aderência ao tratamento clínico. Afinal, trata-se de uma enfermidade praticamente isenta de sintomas, na maioria das vezes diagnosticada por ocasião de um exame oftalmológico rotineiro e que irá exigir terapêutica por um período de tempo indefinido. A isto se deve acrescentar os efeitos colaterais das drogas prescritas, a inconveniência do uso de múltiplas doses de colírios e os custos monetários do tratamento ${ }^{(1)}$. Desta forma, fica fácil entender o porquê dos altos índices de falha na observância do regime terapêutico.

Ao contrário dos tratamentos médicos baseados na ingestão de medi- 
camentos por via oral, o uso correto de colírios depende de uma técnica apropriada. Fraunfelder recomenda que o paciente se deite, deprima a pálpebra inferior com os dedos polegar e indicador para formar um cálice e, em seguida, sem tocar a ponta do frasco no olho, instile uma só gota. Imediatamente após, os olhos devem permanecer fechados por, ao menos, três minutos ${ }^{(2)}$.

Vários estudos demonstraram que o uso incorreto de colírios pode acarretar o aumento da absorção sistêmica das drogas, com conseqüente maior toxicidade das mesmas ${ }^{(3-4)}$. Os efeitos colaterais do maleato de timolol são bastante conhecidos no sistema cardio-pulmonar ${ }^{(5-7)}$ e um estudo recente constatou que $41 \%$ dos pacientes medicados com colírio de brimonidina se queixaram de fadiga e dispnéia aos esforço ${ }^{(8)}$.

Sabe-se que alguns dos mais importantes fatores que levam os pacientes a não aderirem adequadamente ao tratamento do glaucoma podem estar relacionados à instilação incorreta dos colírios. Isto pode favorecer a ocorrência de efeitos colaterais sistêmicos ${ }^{(9)}$, e, também, elevar o custo da terapêutica ${ }^{(10)}$.

Em uma pesquisa onde verificaram a técnica da instilação de colírios em portadores de glaucoma, constatou-se que 18\% dos pacientes estudados utilizaram duas ou mais gotas por instilação e apenas 23\% deles permaneceram com os olhos fechados ou realizaram a oclusão do ponto lacrimal após instilarem $^{(11)}$.

Em um grupo de pacientes portadores de glaucoma instilando colírios foi observado que $45 \%$ deles fizeram contato do bico do frasco com os tecidos oculares. Nenhum dos pacientes estudados manteve o olho fechado por dois minutos ou fez a compressão do ponto lacrimal após a instilação do colírio $^{(12)}$. Além disso, os autores verificaram que foram instiladas uma média de 1,98 \pm 1,87 gotas em cada olho. A constatação, desses autores ${ }^{(12)}$, de que mais de uma gota de medicamento foi utilizada por instilação tem uma importante significância clínica, uma vez que isto pode representar uma maior possibilidade de absorção sistêmica com conseqüentes efeitos colaterais. Este fato pode, também, acarretar uma menor durabilidade do frasco de colírio, com maior custo financeiro do tratamento e menor aderência ao mesmo.

O objetivo do presente trabalho foi observar a técnica de instilação de colírio em uma população de portadores de glaucoma. A finalidade foi detectar possíveis falhas no processo da administração tópica desse medicamento, o que poderia servir de subsídio a uma melhor orientação para o uso correto desta medicação.

\section{MÉTODOS}

A pesquisa foi realizada nos meses de março e abril de 2001 no Ambulatório do Serviço de Glaucoma da Clínica Oftalmológica do Hospital das Clínicas da Faculdade de Medicina da Universidade de São Paulo.

Foram selecionadas as seguintes variáveis demográficas: idade, sexo, raça e escolaridade. Compondo o objeto de estudo, foi introduzida a mensuração do tempo de uso de medicação, o número de colírios utilizados, e a necessidade de outra pessoa para realizar a instilação.

Para tanto, foram recrutados pacientes portadores de qualquer forma de glaucoma crônico que estivessem utilizando pelo menos um colírio hipotensor ocular há mais de um mês. Foram excluídos os pacientes aos quais já houvesse sido explicado, em algum momento, acerca da técnica correta de instilação, bem como os que faziam tratamento há menos de um mês. Sabe-se que de $21 \%$ a 34\% dos pacientes com glaucoma dependem de terceiros para realizar a instilação de seus colírios $^{(1,9)}$. Por esta razão também foram excluídos os pacientes que dependiam rotineiramente de outra pessoa para instilar a medicação, caso esta não estivesse presente no momento do estudo.

Para cada participante do trabalho era fornecido um frasco de colírio de solução lubrificante ocular (Dunason ${ }^{\circledR}$, laboratório Alcon, São Paulo, Brasil), e solicitado que efetuasse a instilação do mesmo, em apenas um olho, escolhido aleatoriamente pelo próprio paciente. Para isso, os pacientes eram conduzidos a uma sala separada, onde havia uma cadeira e uma maca, de forma que pudessem realizar a instilação, tanto quanto possível, da mesma forma que estavam habituados a fazê-lo em seu próprio ambiente. Quando presentes, os acompanhantes que normalmente instilavam a medicação nos pacientes eram, também, instruídos a realizar o mesmo procedimento. O processo era acompanhado por apenas um dos autores, a fim de que a possível tensão de estar sendo observado causasse o mínimo de influência no ato de instilar.

Na instilação, propriamente dita, era verificado o número de gotas utilizadas e o contato ou não da extremidade do frasco de colírio com os tecidos oculares. Era observado se o medicamento atingia ou não a superfície ocular e, caso contrário, se o paciente se dava conta deste fato. Foi verificado se o paciente, após a instilação, fazia a oclusão do ponto lacrimal ou permanecia com o olho fechado por dois minutos. Também, era anotado, se o paciente piscava repetidas vezes logo após o ato da instilação do medicamento.

As variáveis estudadas foram representadas através de média e desvio padrão, mediana e porcentagem. Para análise dos dados obtidos foi utilizado o teste estatístico do Qui quadrado $\left(\chi^{2}\right)$ de Pearson e adotado o nível de significância de $5 \%(0,05)$ para a rejeição da hipótese de nulidade.

\section{RESULTADOS}

No presente trabalho foram estudados 193 pacientes. A idade variou entre 17 a 96 anos, com média de $64 \pm 14,42$ anos e mediana de 66 anos.

Do total de pacientes estudados, 117 (60,7\%) eram do sexo feminino e 76 (39,3\%) do sexo masculino.

Em relação à raça, 87 (45,1\%) dos pacientes eram brancos, 70 (36,3\%) mulatos, 29 (15,0\%) negros e 7 (3,6\%) eram da raça amarela. 
Quanto à escolaridade, 51 (26,4\%) pacientes eram analfabetos, $112(58,0 \%)$ tinham o $1^{\circ}$ grau incompleto, $9(4,7 \%)$ o $1^{\circ}$ grau completo, $4(2,1 \%)$ o $2^{\circ}$ grau incompleto, $13(6,7 \%)$ o $2^{\circ}$ grau completo, e $4(2,1 \%)$ o curso superior (completo ou incompleto).

Os pacientes faziam uso de colírios por um período de tempo que variou entre 2 meses e 40 anos, com média de 7,15 anos e mediana de 4 anos.

Na ocasião do estudo, o número de colírios utilizados variava entre um e quatro, com média de 1,74 $\pm 0,75$ e mediana de um.

Dos 193 pacientes, 93 (48,2\%) faziam uso de apenas um colírio, 66 pacientes $(34,2 \%)$ de dois colírios, 26 pacientes $(13,5 \%)$ de três colírios e 8 pacientes $(4,1 \%)$ de quatro colírios.

Quanto à posição de instilar a medicação, 101 (52,3\%) pacientes ficaram sentados, 50 (25,9\%) permaneceram em pé e 42 (21,8\%) preferiram deitar-se.

Constatou-se que 23 pacientes (11,9\%) utilizaram-se de uma terceira pessoa para proceder à instilação. Desses, 9 (4,7\%) o fizeram por alguma deficiência física e 14 (7,2\%) pacientes por comodismo, inabilidade ou falta de confiança.

Na instilação propriamente dita, o número de gotas utilizadas variou de zero a dez, com média de 1,64 \pm 1,26 gotas e mediana de uma gota. Quando a instilação era realizada pelo próprio paciente, em média foram utilizadas $1,71 \pm 1,32$ gotas. Por outro lado, quando o colírio foi instilado por terceiros, foram empregadas em média 1,13 \pm 0,54 gotas.

A tabela 1 correlaciona os diferentes períodos de tempo de tratamento com a medicação tópica em relação ao número de gotas utilizadas e o contato ou não da extremidade do frasco de colírio com os tecidos oculares. Ela permite observar que não houve significância estatística entre o tempo de tratamento e o número de gotas utilizadas $\left(\chi^{2}=7,85 \mathrm{p}=0,097\right)$, assim como entre o tempo de tratamento e o fato de fazer ou não contato do frasco de colírio com a superfície ocular $\left(\chi^{2}=2,06\right.$ $\mathrm{p}=0,356)$.

A tabela 2 correlaciona a escolaridade do grupo estudado com o contato do bico do frasco de colírio com a superfície ocular, com a compressão ou não do ponto lacrimal (ou a manutenção do olho fechado por dois minutos), e com o ato ou não de piscar após a instilação. Ela mostra que não houve correlação estatisticamente significante entre a escolaridade e o fato de fazer ou não contato do frasco de colírio com a superfície ocular $\left(\chi^{2}=2,97 \mathrm{p}=0,226\right)$, em realizar ou não a compressão do ponto lacrimal $\left(\chi^{2}=2,98 \mathrm{p}=0,225\right)$, e entre o piscar repetidamente ou não logo após ter instilado $\left(\chi^{2}=3,54 \mathrm{p}=0,170\right)$.

A tabela 3 correlaciona os diferentes períodos de tempo de tratamento tópico com a compressão ou não do ponto lacrimal (ou manutenção do olho fechado por dois minutos), imediatamente após a instilação. Ela demonstra que não houve significância estatística entre estes itens $\left(\chi^{2}=0,36 p=0,835\right)$.

A tabela 4 correlaciona o contato ou não da extremidade do frasco de colírio com a superfície ocular e o ato de piscar repetidamente logo após a instilação, observando-se haver relação estatisticamente significativa entre eles $\left(\chi^{2}=4,04\right.$ $\left.\mathrm{p}=0,04454^{*}\right)$.

Em 6 casos (3,1\%) o medicamento não atingiu a superfície ocular, sendo que os pacientes não se deram conta do fato. Destes, em 5 casos, o paciente instilou o colírio sozinho e, em um caso, a instilação foi realizada por uma outra pessoa.

\section{DISCUSSÃO}

O sucesso do tratamento clínico do glaucoma crônico é baseado no uso de drogas que visam reduzir a pressão intraocular. A fim de que tal objetivo seja alcançado utilizam-se medicações administradas, na maioria das vezes, sob a forma de colírios.

Para o paciente portador de glaucoma é preciso não somente prescrever o medicamento apropriado como, também, orientá-lo quanto à sua utilização. Para tanto, é fundamental que o oftalmologista ensine a seu paciente a técnica correta da instilação de colírios.

O presente trabalho verificou que a maioria dos pacientes estudados usou de modo inadequado esse tipo de medicação. Em média, eles utilizaram 1,64 \pm 1,26 gotas por instilação e a tabela 1 mostra que cerca de 74 (38,3\%) dos pacientes usaram duas ou mais gotas e que não houve correlação estatisticamente significante entre o número de gotas utilizadas e o tempo de tratamento do glaucoma. Ou seja, não é porque se trata a mais tempo que se usa o número adequado de gotas.

Alguns pacientes utilizam propositadamente mais de uma gota de colírio com o intuito de obter um efeito hipotensor ocular mais eficiente ${ }^{(3)}$.

Normalmente, o olho humano pode acomodar uma gota de colírio com volume não superior a $23 \mu \mathrm{l}^{(4,13)}$. Quantidades superiores a esta escorrem pela região facial e são desperdiçadas, ou são drenadas pela via lacrimal com possibilidade de maior absorção sistêmica. Na realidade, esta absorção através da mucosa rinofaríngea leva a efeitos farmacológicos comparáveis a uma injeção endovenosa, tal a sua rapidez de aparecimento ${ }^{(4)}$.

Por outro lado, o volume das gotas dos colírios para glaucoma, comercialmente disponíveis, variam de $31 \mu \mathrm{l}$ a $61 \mu \mathrm{l}^{(14-15)}$. Isto, não somente significa um maior desperdício, bem como a possibilidade de uma maior absorção sistêmica com conseqüente maior efeito colateral. Devemos, também, realçar que o maior desperdício está associado com o aumento do custo da terapêutica, fato fundamental em se tratando de uma doença crônica, numa população com características socioeconômicas desfavoráveis.

Quanto à constatação de que 50 (25,9\%) indivíduos medicaram-se em pé, deve-se lembrar que esta posição pode tornar mais difícil ao paciente manter os olhos fechados por dois minutos logo após a instilação. A compressão do ponto lacrimal ou a simples manutenção dos olhos fechados durante dois minutos imediatamente após o uso da medicação reduz, significativamente, a absorção sistêmica das drogas e aumenta de forma expressiva a penetração da mesma na câmara anterior ${ }^{(16)}$. 


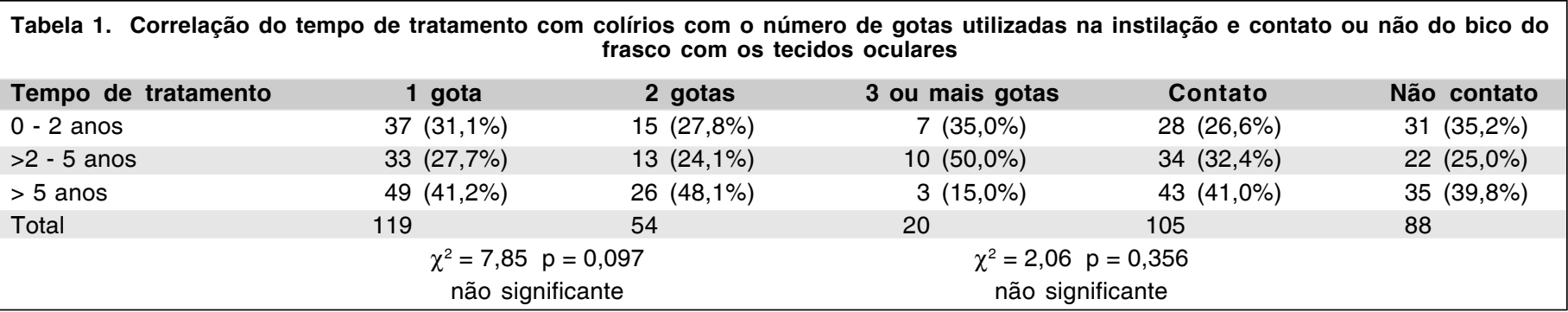

\begin{tabular}{|c|c|c|c|c|c|c|}
\hline Escolaridade & Contato & Não contato & Compressão & Não compressão & Piscar & Não piscar \\
\hline Analfabeto & $33(31,4 \%)$ & $18(20,5 \%)$ & $10(40,0 \%)$ & $41(24,4 \%)$ & $34(28,6 \%)$ & $17(23,0 \%)$ \\
\hline $1^{\circ} \mathrm{grau}$ incompleto & $57(54,3 \%)$ & $55(62,5 \%)$ & $11(44,0 \%)$ & $101(60,1 \%)$ & $71(59,7 \%)$ & $41(55,4 \%)$ \\
\hline $1^{\circ}$ grau completo ou mais & $15(14,3 \%)$ & $15(17,0 \%)$ & $4(16,0 \%)$ & $26(15,5 \%)$ & $14(11,7 \%)$ & $16(21,6 \%)$ \\
\hline \multirow[t]{2}{*}{ Total } & 105 & 88 & 25 & 168 & 119 & 74 \\
\hline & \multicolumn{2}{|c|}{$\begin{array}{c}\chi^{2}=2,97 p=0,226 \\
\text { não significante }\end{array}$} & \multicolumn{2}{|c|}{$\begin{array}{c}\chi^{2}=2,98 \quad p=0,225 \\
\text { não significante }\end{array}$} & \multicolumn{2}{|c|}{$\begin{array}{c}\chi^{2}=3,54 \mathrm{p}=0,170 \\
\text { não significante }\end{array}$} \\
\hline
\end{tabular}

\begin{tabular}{|c|c|c|c|}
\hline $\begin{array}{l}\text { Tempo de } \\
\text { tratamento }\end{array}$ & Compressão & Não compressão & Total \\
\hline $0-2$ anos & $8(32,0 \%)$ & $51(30,4 \%)$ & 59 \\
\hline$>2-5$ anos & $6(24,0 \%)$ & $50(29,8 \%)$ & 56 \\
\hline$>5$ anos & $11(44,0 \%)$ & $67(39,8 \%)$ & 78 \\
\hline Total & 25 & 168 & 193 \\
\hline
\end{tabular}

\begin{tabular}{|l|lcr|}
\hline \multicolumn{4}{|c|}{$\begin{array}{l}\text { Tabela 4. Correlação entre o contato ou não da extremidade do } \\
\text { frasco de colírio com a superfície ocular e o ato de piscar após a } \\
\text { instilação }\end{array}$} \\
Ato de piscar & $\begin{array}{c}\text { Não contato } \\
\text { do frasco }\end{array}$ & $\begin{array}{c}\text { Contato } \\
\text { do frasco }\end{array}$ & Total \\
Sim & $\begin{array}{l}47(53,4 \%) \\
72(68,6 \%)\end{array}$ & $119(61,7 \%)$ \\
Não & $41(46,6 \%)$ & $33(31,4 \%)$ & $74(38,3 \%)$ \\
Total & 88 & 105 & 193 \\
& \multicolumn{4}{c}{$\chi^{2}=4,04 \quad p=0,04454^{*}$} \\
significante
\end{tabular}

Alguns autores recomendam orientar o paciente para que instile o colírio sentado ou deitado e feche suavemente os olhos logo após a instilação, mantendo-os cerrados por cerca de dois minutos ${ }^{(1)}$. Este simples fato, segundo os autores, além de produzir os efeitos acima citados, pode levar ao aumento da aderência ao tratamento do glaucoma.

A tabela 1, também, verificou que cerca de 105 (54,4\%) dos pacientes estudados fizeram contato entre a extremidade do frasco de colírio e a superfície ocular. Sabe-se que o contato do bico do frasco da medicação com a superfície ocular favorece a contaminação de seu conteúdo pela flora microbiana $\operatorname{ocular}^{(2)}$. Além do mais, esse contato pode levar à sucção do filme lacrimal para dentro do frasco de colírio, alterando as propriedades farmacológicas da droga em questão ${ }^{(3)}$.

A sucção da lágrima para dentro do frasco de colírio de pilocarpina leva à transformação dessa droga em isopilocarpina, a qual não possui qualquer propriedade hipotensora ocular ${ }^{(3)}$.

Outros dois fatores adversos do contato do frasco com a superfície ocular são o aumento da freqüência do piscar e do lacrimejamento reflexo ${ }^{(2)}$. Isto dilui a droga e aumenta sua velocidade de drenagem através da via lacrimal acarretando, respectivamente, a menor concentração farmacológica da droga na câmara anterior e a maior absorção sistêmica da mesma via mucosa rinofaríngea ${ }^{(4)}$.

A tabela 4 mostra que 105 (54,4\%) dos pacientes estudados fizeram contato da extremidade do frasco com a superfície ocular e que tal fato aumentou substancialmente a freqüência do ato de piscar nessa população.

As tabelas 2 e 3 mostram que 168 (87\%) dos pacientes estudados não fez a oclusão do ponto lacrimal (ou manteve o olho fechado por dois minutos), e que cerca de 119 (62\%) dos pacientes piscaram repetidamente logo após a instilação do colírio, não havendo correlação, estatisticamente significante, entre este último dado e o tempo de tratamento e a escolaridade da população estudada.

Estudos anteriores constataram que 25\% - 57\% dos pacientes com glaucoma tem dificuldades para instilar sozinhos o colírio prescrito, sendo que $21 \%$ deles dependem rotineiramente de terceiros para usar esta medicação ${ }^{(10,17)}$.

No presente trabalho, em 23 (11,9\%) dos pacientes estudados, uma outra pessoa aplicou a medicação. Este número, inferior aos dados da literatura, provavelmente deve-se ao 
fato de que esta categoria de pacientes foi incluída em nossa pesquisa apenas quando o acompanhante que instila a medicação estava presente no momento do estudo. Desses 23 pacientes 9 eram portadores de deficiência física e 14 não se sentiam confortáveis em se automedicar. É interessante notar que, quando a instilação foi realizada por terceiros, empregouse, em média, 1,13 $\pm 0,54$ gotas, enquanto que, quando o colírio foi instilado pelo próprio paciente, o número de gotas utilizadas foi de $1,71 \pm 1,32$.

Esse fato reforça as dificuldades de um regime terapêutico com múltiplas doses diárias, especialmente para esse tipo de paciente.

No estudo atual observamos que, em seis casos, o colírio instilado não atingiu a superfície ocular e os pacientes não se deram conta desse fato. Este acontecimento, embora surpreendente, já foi constatado por outros autores e pode estar associado à redução da capacidade cognitiva de certos pacientes mais idosos ${ }^{(9,18)}$.

Deve-se ressaltar que, em um caso dos seis acima citados, a instilação foi realizada por outra pessoa, demonstrando possível inabilidade para uma ação aparentemente tão simples.

Concluindo, foi observado na população estudada uma grande deficiência técnica no aparente simples ato de instilar um colírio.

Este fato acarretou o desperdício de parte do conteúdo do frasco, a contaminação da extremidade do mesmo, o aumento da possibilidade de toxicidade sistêmica e a possível diminuição da concentração da droga na câmara anterior.

O glaucoma é uma doença crônica e o seu controle depende do uso adequado da medicação prescrita, sendo que muitos dos nossos pacientes não controlados não tem sucesso terapêutico devido à técnica incorreta da instilação dos seus colírios.

Infelizmente, muitos dos doentes que evoluem com alteração progressiva do campo visual poderiam ter um prognóstico bem menos sombrio se fossem melhor orientados no ato, aparentemente banal, de instilar colírios nos olhos.

Finalizando, ao nosso ver, é fundamental o ensino da técnica adequada da instilação de colírios mesmo para o grupo de pacientes que utilizam essa medicação há muitos anos, independente da sua escolaridade e do seu nível socioeconômico.

\section{ABSTRACT}

Purpose: To observe eye drop instillation technique in chronic glaucoma patients. Methods: This is a prospective study enrolling 193 glaucomatous patients. Each received a sample of lubricant eye drop bottle (Dunason ${ }^{\circledR}$, Alcon Laboratories, São Paulo, Brazil) and was instructed to use it once. Results: All patients used a mean amount of $1.64 \pm 1.26$ drops. In $54.5 \%$ of the patients, the eyedropper touched the eye and adnexa. In $3.1 \%$ the drops were not placed in the eye, without the patient being aware of this. In $87.0 \%$ of them, neither the lacrimal point occlusion nor the closure of the eyes for two minutes after instillation were observed, and $61.6 \%$ repeatedly blinked right after instilling the drop. Conclusions: Most patients did not use the eye drops properly. That may result in some undesirable consequences, such as, a partial wastage of the bottle's content, a possible increase in systemic toxicity, the risk of contamination of the bottletip, and, mainly, a decrease in the full hypotensive effect of the drugs. Therefore, the patients have to be carefully taught the correct way to use eye drops.

Keywords: Glaucoma/therapy; Instillation, drug; Prescription drugs

\section{REFERÊNCIAS}

1. Zimmerman TJ, Zalta AH. Facilitating patient compliance in glaucoma therapy. Surv Ophthalmol 1983;28(Suppl):252-8.

2. Fraunfelder FT. Extraocular fluid dynamics: how best to apply topical ocular medication. Trans Am Ophthalmol Soc 1976;74:457-87.

3. File RR, Patton TF. Topically applied pilocarpine. Human pupillary response as a function of drop size. Arch Ophthalmol 1980;98:112-5.

4. Shell JW. Pharmacokinetics of topically applied ophthalmic drugs. Surv Ophthalmol 1982;26:207-18.

5. Hayreh SS, Podhajsky P, Zimmerman MB. Beta-blocker eyedrops and nocturnal arterial hypotension. Am J Ophthalmol 1999;128: 301-9.

6. Avorn J, Glynn P, Gurwitz J. Pulmonary effects of beta-blockers. J Glaucoma 1993;2:158-65.

7. Van Buskirk EM, Fraunfelder FT. Ocular beta-blockers and systemic effects. Am J Ophthalmol 1984;98:623-4.

8. Waldock A, Snape J, Grabam CM. Effects of glaucoma medications on the cardiorespiratory and intraocular pressure status of newly diagnosed glaucoma patients. Br J Ophthalmol 2000;84:710-3.

9. Winfield AJ, Jessiman D, Williams A, Esakowitz L. A study of the causes of non-compliance by patients prescribed eyedrops. Br J Ophthalmol 1990;74: 477-80.

10. Pedroso L, Carvalho Jr ES, Paranhos Jr A, Prata Jr JA, Mello PAA. Custo real do tratamento de glaucoma para o paciente. Arq Bras Oftalmol 1999; 62:677-82.

11. Costa VP, Vasconcellos JPC, Pelegrino M, Kara-José N. Análise da técnica de instilação de colírios por pacientes glaucomatosos. Rev Bras Oftalmol 1995;54:523-8.

12. Kass MA, Hodapp E, Gordon M, Kolker A E, Goldberg I. Patient administration of eyedrops: observation. Part II. Ann Ophthalmol 1982,14:889-93.

13. Nagataki S, Mishima S. Pharmacokinetics of instilled drugs in the human eye. Int Ophthalmol Clin 1980;20:33-49.

14. Stewart WC, Sine C, Cate E, Minno GE, Hunt HH. Daily cost of betaadrenergic blocker therapy. Arch Ophthalmol 1997;115:853-6.

15. Amaral Fo JM, Moreira RAR, Silva LMS, Vasconcelos JP, Rocha EM, Costa VP, Kara-José N. Custo mensal de medicações antiglaucomatosas no Brasil. Arq Bras Oftalmol 1999;62:123-6.

16. Zimmerman TJ, Kooner KS, Kandarakis AS, Ziegler LP. Improving the therapeutic index of topically applied ocular drugs. Arch Ophthalmol 1984; 102:551-3.

17. Vincent PA. Patients' viewpoint of glaucoma therapy. Sight Sav Rev 1972; 42:213-21.

18. Morrow D, Leirer V, Sheikh J. Adherence and medication instructions. Review and recommendations. J Am Geriatr Soc 1988;36:1147-60. 